\section{Reflexiones en torno a las prácticas educativas del Diploma de Operador Socioeducativo en Economía Social y Solidaria en clave de Investigación Acción Participativa}

\author{
Luciano Petit \\ Docente investigador de la Universidad \\ Nacional de Quilmes, Argentina. \\ Mónica Copello \\ Docente y miembro del Programa \\ de Extensión Universitaria y de \\ Diploma de Operador Socioeducativo \\ en Economía Social y Solidaria de la \\ Universidad Nacional de Quilmes
}

Nacional de Quilmes.

\section{Yolanda Bianchi}

Miembro del Programa de Extensión

Universitaria y del Diploma de

Operador Socioeducativo en Economía

Social y Solidaria de la Universidad

\section{Resumen}

En el presente artículo pretendemos contar la experiencia del curso de extensión universitaria "Universidad y Economía Social y Solidaria. Reflexionando las prácticas educativas del Diploma de Operador Socioeducativo en Economía Social y Solidaria en clave de Investigación Acción Participativa" de la Universidad Nacional de Quilmes (Bernal, Argentina) como propuesta de reflexión sobre las prácticas para fortalecerlas, práctica-teoría-práctica, desde el método de la investigación acción participativa, cuyo objetivo es investigar desde y para las prácticas educativas de la Economía Social y Solidaria (ESS). Las acciones pretendidas se enmarcan en el modelo dialógico de la pedagogía a partir de las comunidades de aprendizaje como procesos educativos de inclusión y transformación, pues pensamos a la ESS como un proyecto de transformación social y política, que implica otras formas de hacer y pensar la economía pero también otras formas de hacer y pensar la educación. Concepción política pedagógica coherente con la búsqueda de un sujeto crítico, en un colectivo que analiza y actúa sobre su entorno desde los valores de autogestión, asociativismo y solidaridad.

\section{Palavras chave}

- Economía Social y Solidaria

- Diploma

- extensión universitaria

- investigación acción participativa
Economía Social y Solidaria /

Intervenciones

RECEPCIÓN: 26/06/15

ACEPTACIÓN FINAL: 02/09/15

\section{Resumo}

No presente artigo procuramos relatar a experiência do curso de extensão universitária "Universidade e Economia Social e Solidária. Refletindo as práticas educacionais do Diploma de Operador Socioeducativo em Economia Social e Solidária na chave de Pesquisa Ação Participativa" da Universidade Nacional de Quilmes (Bernal, Argentina) como proposta de reflexão sobre as práticas para fortalecê-las, prática-teoria-prática, do método da pesquisa ação participativa, cujo objetivo é pesquisar desde e para as práticas educacionais da Economia Social e Solidária (ESS). Emoldurando as supostas ações no modelo dialógico da pedagogia a partir das comunidades de aprendizagem como procesos educativos de inclusão e transformação pois achamos à ESS como um projeto de transformação social e política, envolvendo outras formas de fazer e pensar a economia, mas também outras formas de fazer e pensar o ensino. Concepção política pedagógica coerente com a procura de um sujeito crítico, em um grupo que analisa e atúa em seu ambiente a partir de valores de autogestão, associações e solidariedade

Palabras-clave

- Economia Social e Solidária

- Diploma

- extensão universitária

- pesquisa ação participativa
Para citación de este artículo

Petit, L.; Copello, M. y Bianchi, Y. (2015). Reflexiones en torno a las prácticas educativas del Diploma de Operador Socioeducativo en Economía Social y Solidaria en clave de Investigación Acción Participativa. En Revista +E versión digital, (5), pp. 168-173. Santa Fe, Argentina: Ediciones UNL. 


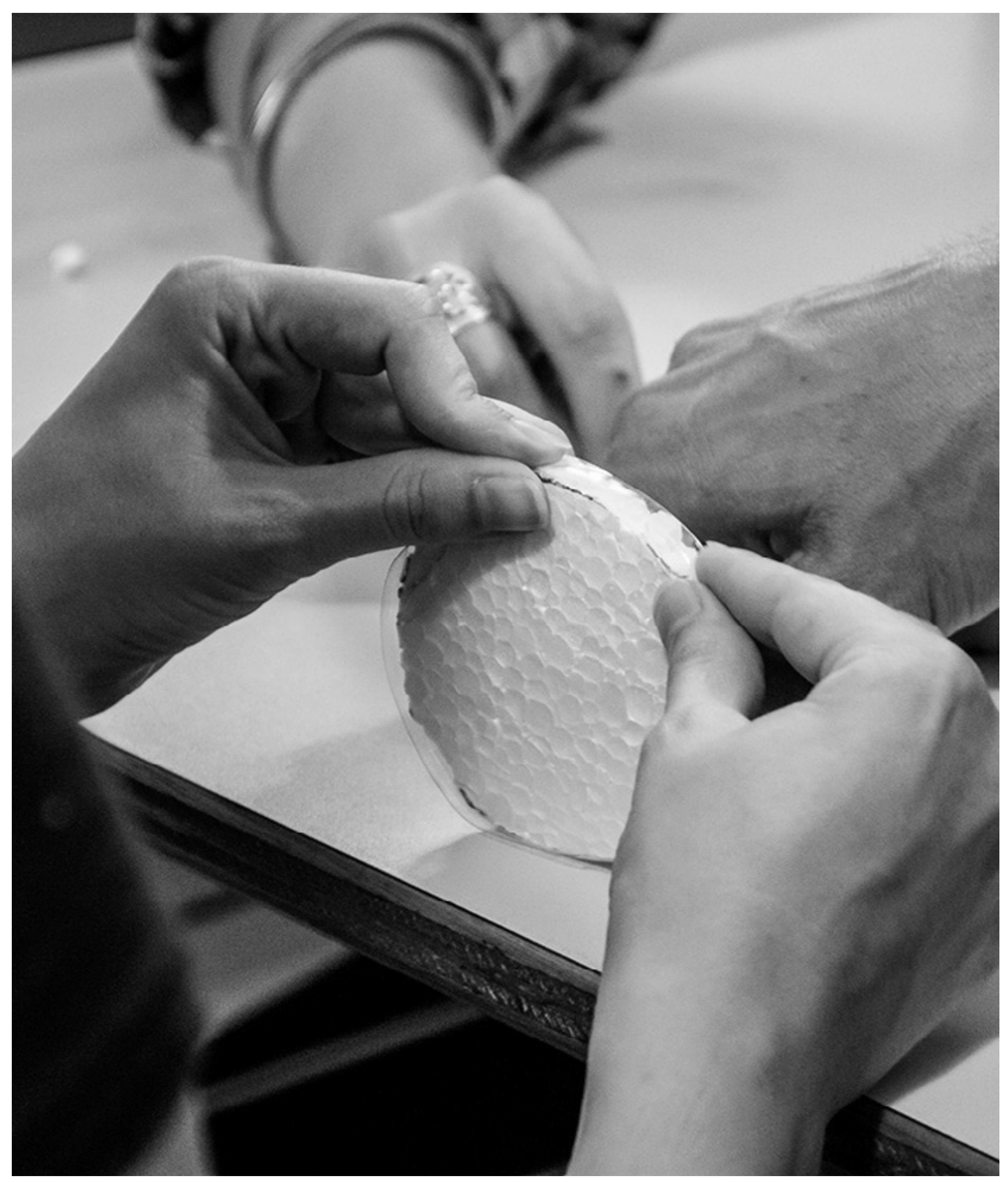

Reflexiones en torno a las prácticas... I Petit, L.; Copello, M. y Bianchi, Y.। 169 


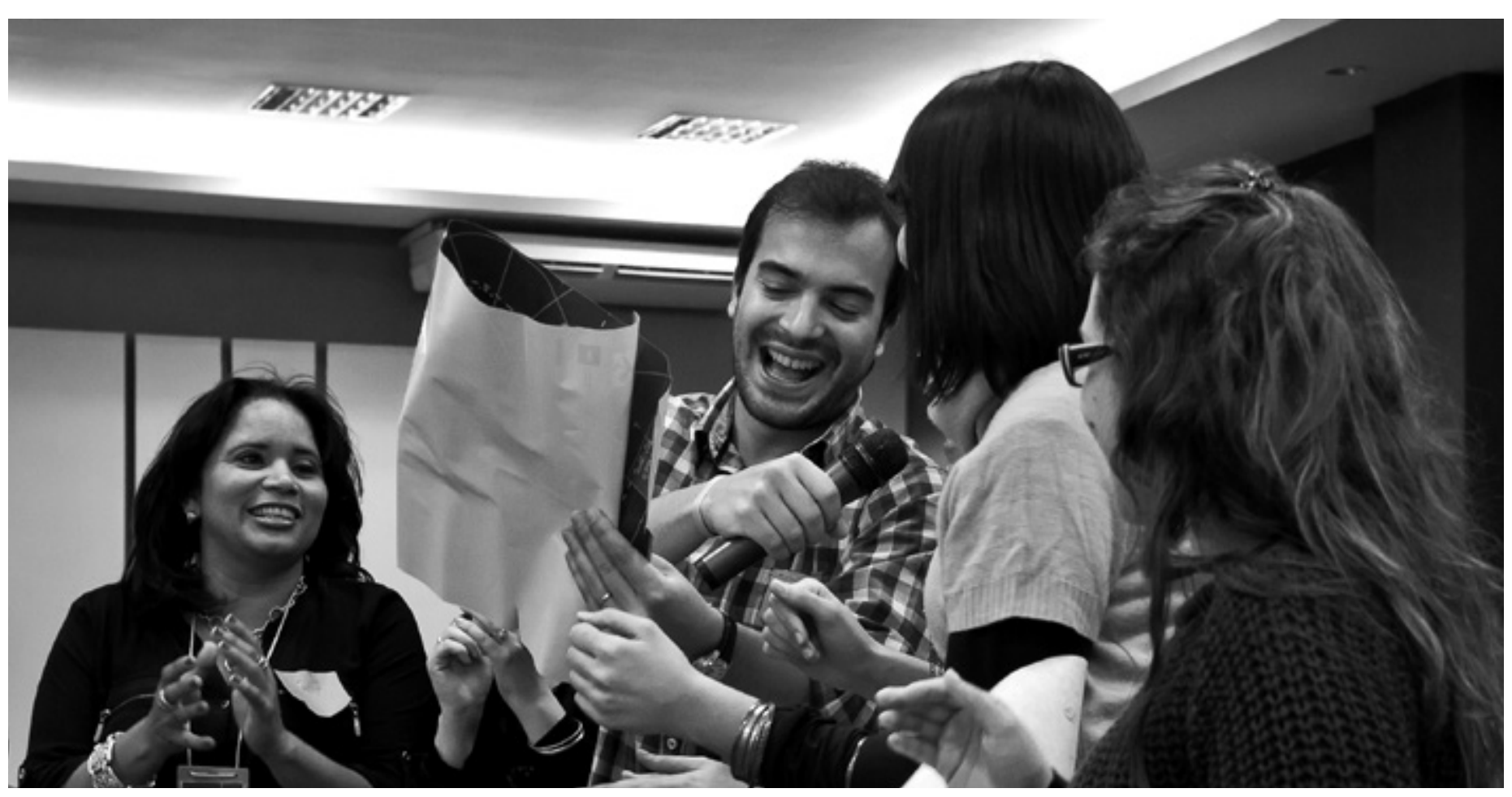

\section{Introducción}

En el presente artículo pretendemos contar la experiencia del curso de extensión universitaria "Universidad y Economía Social y Solidaria. Reflexionando las prácticas educativas del Diploma de Operador Socioeducativo en Economía Social y Solidaria en clave de Investigación Acción Participativa" como propuesta de reflexión sobre las prácticas para fortalecerlas, práctica-teoría-práctica, desde el método de la investigación acción participativa, cuyo objetivo es investigar desde y para las prácticas educativas de la Economía Social y Solidaria (ESS). Las acciones pretendidas se enmarcan en el modelo dialógico de la pedagogía a partir de las comunidades de aprendizaje (Ferrada y Flecha, 2008) como procesos educativos de inclusión y transformación pues pensamos a la ESS como un proyecto de transformación social y política, que implica otras formas de hacer y pensar la economía, pero también otras formas de hacer y pensar la educación.

Iniciado en 2013 , el curso de extensión universitaria pretende reflexionar sobre las prácticas educativas y la propuesta pedagógica del Diploma de Operador Socioeducativo en Economía Social y Solidaria (DOSESS) a partir de la aplicación de la metodología de Investigación Acción Participativa con respecto a la relación entre Universidad y Economía Social y Solidaria. El curso se vincula sinérgicamente con el Proyecto de Extensión Construyendo Redes Emprendedoras de Economía Social (CREES); el DOSESS:
Formación y prácticas socioeducativas para el fortalecimiento de los actores de la ESS; el Proyecto de Voluntariado Universitario "Formación en Economía Social y Solidaria en la Universidad Nacional de Quilmes" y el Proyecto de Investigación "Economía social y procesos educativos y de salud: algunos estudios de casos de cooperación social"; del cual participan diplomados de este trayecto formativo, parte del equipo docente y la coordinación. El curso se planteó tres objetivos específicos:

1) analizar la experiencia del DOSESS en tanto propuesta pedagógica para la construcción y el fortalecimiento de la ESS; 2) visualizar la importancia de la sistematización como parte del proceso de evaluación de las prácticas de ESS; y

3) trabajar sobre la construcción del rol del operador socioeducativo en Economía Social y Solidaria.

Los contenidos del mismo se articularon con relación a tres ejes: A) el DOSESS en tanto propuesta pedagógica para la construcción y el fortalecimiento de la ESS;

B) la sistematización como herramienta de evaluación en las prácticas educativas en ESS; y

C) la construcción del rol de operador socioeducativo en Economía Social y Solidaria en el territorio; las prácticas profesionalizantes como espacio de aprendizaje. 
Cabe destacar la articulación entre extensión e investigación, pues el proyecto de investigación se propone aportar conocimientos sobre la articulación existente entre la economía solidaria y los procesos educativos analizando en particular algunas experiencias relevantes; específicamente, la sistematización y análisis de la experiencia del Diploma de Extensión Universitaria en Economía Social y Solidaria.

\section{Diploma de Operador Socioeducativo en Economía Social y Solidaria}

Este Diploma forma parte de la labor que se desarrolla en el área de extensión de la Universidad Nacional de Quilmes (UNQ) en torno a la temática de ESS. Específicamente, es una de las actividades del Proyecto de Extensión Universitaria CREES-DOSESS: Formación y prácticas socioeducativas para el fortalecimiento de los actores de la ESS que integra el Programa CREES-ICOTEA (Integración, Comunidad de Aprendizaje, Tecnologías para la inclusión social, Empresa social, Arte comunitario).

Este Programa surge como iniciativa superadora del entonces proyecto CREES, el cual venía trabajando en ESS desde el año 2006. A partir de 2011, cuando el Consejo Superior de la UNQ aprueba la conformación de los "Programas de Extensión Universitaria", nos proponemos el desafío de conformar el CREES-ICOTEA y aunamos siete proyectos originarios, nueve en la actualidad y en distinta etapa evolutiva, entre los cuales se articula desde la extensión.

Si bien la denominación ICOTEA es una sigla que hace alusión a los proyectos que conforman el Programa, también es una alegoría tomada de las ideas del sociólogo colombiano Orlando Fals Borda (1993), quien en sus estudios sobre la cultura ribereña en la costa del Mar Caribe menciona al "hombre hicotea", en quien reconoce las características de la tortuga homónima (Hicotea o Jicotea). La persona "hicotea" es un ser sentipensante, resistente para poder afrontar y superar las adversidades de la cotidianeidad, que despliega la capacidad de esperar su tiempo, protegiéndose ante los contratiempos con su caparazón para luego volver a la realidad con la misma fortaleza de antes; pero además no abandona su capacidad de disfrute y alegría a pesar de los percances que puedan afligirla. Así es como nos movemos en la ESS: hay que tener destrezas para desarrollar actividades económicas con objetivos sociales, y actividades sociales con objetivos económicos.

Con esta perspectiva elaboramos el DOSESS, un trayecto integral de extensión universitaria compuesto por cinco materias (Economía Social y Solidaria, Trabajo y Sociedad; Grupos, Organizaciones y Equipos de Trabajo, Educación Social y Comunidad de Aprendizaje; y Tecnologías de la Información y la Comunicación) y un seminario de prácticas profesionalizantes, que suman en total una carga horaria de 500 horas (320 horas áulicas y 180 de horas prácticas). Esta propuesta formativa se fundamenta en una metodología educativa sustentada en la experiencia de la educación popular. Es comprendida como un proceso que propicia la construcción colectiva de conocimiento, en la que se valoran los saberes de todos los participantes, desterrando la visión del monopolio del conocimiento por el docente y valorando a la persona en su integralidad.

Desde su creación en 2010, se han desarrollado dos cohortes en articulación con los ministerios de Desarrollo Social y Educación y otras universidades. Asimismo, en el marco del vínculo con las organizaciones de la zona se conformaron cinco comisiones.

La primera experiencia, articulada con los ministerios, en números se sintetiza en: 25 comisiones distribuidas en 14 sedes en el Conurbano Bonaerense; un equipo de coordinación interinstitucional ${ }^{1}$; tres coordinaciones regionales; cinco coordinadores de espacios curriculares; 120 docentes incorporados a la experiencia trabajando en cada aula como pareja pedagógica y conformando equipos docentes regionales; 872 estudiantes inscriptos de los cuales el 80 $\%$ concluyó la cursada, de los cuales el 96 \% se graduó y el resto tiene pendiente alguna instancia de evaluación.

La segunda se inició en octubre de 2012 y reflejada en números es: 22 comisiones distribuidas en 9 sedes en el Conurbano Bonaerense; 1 equipo de coordinación general (integrado por 1 coordinador general, 2 coordinadores adjuntos, 1 coordinadora académica y de prácticas y 1 asistente ejecutivo); 3 coordinaciones regionales; 4 coordinadores de espacios curriculares y 2 colaboradoras en coordinación curricular; 140 docentes trabajando en cada aula como pareja pedagógica y conformando equipos docentes regionales; 478 estudiantes inscriptos de los cuales el $81 \%$ concluyó la cursada, de los cuales el 84 \% se graduó y el resto tiene pendiente alguna instancia de evaluación.

Respecto a la cohorte abierta, desde 2012 a la fecha, se han abierto 5 comisiones, en la actualidad no contamos con información detallada de las mismas pues los datos se encuentran en fase de procesamiento.

Aprobado por resolución del Consejo Superior $N^{\circ} 176 / 10$, el DOSESS es parte de una estrategia educativa que tiene como objetivos incentivar la terminalidad de la educación media y el ingreso y permanencia en la Universidad u otros espacios educativos. En este sentido, pensando en su continuidad, se ha creado en esta Universidad, la Tecnicatura Universitaria en Economía Social y Solidaria.

1) Integrado por un coordinador general, dos coordinadores adjuntos y una coordinadora académica y de prácticas. 
A través del enfoque de la educación social y popular, se revalorizan las trayectorias de aprendizaje vital habilitadas por el diálogo de saberes y la co-construcción de conocimiento. Esta concepción política pedagógica es coherente con nuestra visión alternativa de la economía, ya que busca el desarrollo de un sujeto crítico en un colectivo que analiza y actúa sobre su entorno desde los valores de autogestión, asociativismo y solidaridad (Petit y Roura, 2013).

Es decir, un operador socioeducativo en ESS se constituye como un sujeto con capacidad de reflexión sobre sus acciones y prácticas, con herramientas conceptuales que le posibilitan diseñar y construir nuevas experiencias vinculadas a la ESS, transformando los preexistentes, $y$, además, puede multiplicar los procesos de desarrollo educativo y comunitario en sus espacios territoriales. (Petit, Baliño, Iglesias y Garo, 2014).

\section{Investigación Acción Participativa como método para reflexionar sobre las prácticas educativas del DOSESS}

El método de investigación acción participativa es el indicado cuando el investigador se propone no únicamente conocer la realidad específica de un grupo sino que además desea actuar. A partir de lo cual los sujetos investigados participan en el rol de coinvestigadores en todas las etapas del proceso de indagación. Siendo la finalidad central de estas investigaciones orientar la concientización, desarrollo y emancipación de los grupos observados y hacia la transformación (Martínez, 2006).

En decir, es el estudio de un contexto social a partir del proceso de pasos encadenados en progresión a partir de lo cual se investiga y simultáneamente se interviene (Montero y León, 2007). Se centra en la acción, se involucra y compromete en realidades concretas, estableciendo un dialogo entre los saberes académicos y los saberes populares.

Para nuestro trabajo se utilizó una metodología cualitativa y se diagramó un plan de investigación (método o diseño) de estudio de caso, múltiple (Montero y León, 2007), siendo un estudio descriptivo no estructurado que tiene una única unidad muestral: el grupo participante del encuentro. Por ello, los investigadores eligieron dos casos ejemplificadores que presentan variantes. Se analizaron como casos dos cursos del programa de extensión universitaria "Universidad y Economía Social y Solidaria. Reflexionando las prácticas educativas del Diploma de Operador Socioeducativo en Economía Social y Solidaria en clave de Investigación Acción Participativa" realizados en la Universidad Nacional de Quilmes, Bernal (Argentina).

El primer curso se hizo en el año 2013, desde el mes de julio a noviembre, el primer viernes de cada mes. En promedio, asistieron a cada encuentro 50 estudiantes de distintos territorios del Conurbano Bonaerense y 6 docentes.
Se asumió el desafío de pensar, debatir, sistematizar y escribir de manera colectiva las diferentes experiencias con el objeto de mejorar las presentes y futuras prácticas formativas para darlas a conocer a otros actores sociales y experiencias colectivas. Cada jornada comenzó con una dinámica de bienvenida, que celebró la posibilidad de juntarnos a compartir un espacio y un tiempo con el objetivo de disfrutar el conocernos y re conocernos para reflexionar sobre nuestras prácticas cotidianas. Luego una breve exposición conceptual daba el marco para posteriormente, en un segundo momento, asignarle a cada grupo una consigna generadora de intercambios y construcciones colectivas de conocimientos nuevos. Finalmente, se trabajaba en la escritura de los acuerdos y de una síntesis en cada grupo.

Si comparamos la edición 2013 de este curso con la realizada en el año 2014, podemos observar que cambiaron la estructura, los participantes y el contenido.

Una herramienta que fue adquiriendo relevancia y protagonismo, fue la música que en el primer curso resultara un emergente para transformarse en el segundo en un contenido planificado por el equipo docente. Luego, el contenido música generó el emergente del encuentro sentipensante a partir de las historias de vida que las canciones invitaban a compartir, de los ritmos que movilizaban los cuerpos hacia otro desplazamiento en el espacio físico, de la alegría que producía el encontrar letras y melodías en común, temas que habían marcado algún período de nuestras vidas. En la primera edición del curso, la música como emergente quedó plasmada en una producción que utilizando la melodía de "EI mundo del revés" de María Elena Walsh nos permitió abordar distintos contenidos curriculares y sintetizarlos en una letra que llevó por título "El mundo de la ESS".

En la segunda edición de 2014, en función del tipo de música que, más allá de lo planificado, surgía insistentemente en cada encuentro, se compuso un "Chamamess o el chamamé de las prácticas en ESS" en el que se desarrollaban los contenidos curriculares correspondientes a la materia Prácticas Profesionalizantes. Las dos canciones tuvieron su instancia de grabación para lo cual hubo que vincularse con otras áreas de la Universidad, como Auditorio, EPA CREES (Equipo de Producción Audiovisual de nuestro Programa de Extensión); docentes y estudiantes del Diploma y la Tecnicatura para poder contar con los recursos y las voces e instrumentación necesarias.

La metodología para llevar adelante el proceso de escritura fue la de trabajar en pequeños grupos los contenidos: pareja pedagógica, comunidad de aprendizaje, contenidos curriculares, prácticas profesionalizantes y rol del operador socio educativo en ESS. Lo redactado por un grupo en un encuentro rotaba para ser leído y reelaborado por otro y así sucesivamente hasta llegar a una versión final por cada ítem, material con el cual se hicieron cartillas de difusión para las próximas cohortes del Diploma. 


\section{Reflexión final}

La dicotomía es una realidad que no está exenta del ámbito universitario y pareciera ser constitutiva de la vida académica. Uno de sus versus más tradicionales es la diferencia entre extensión e investigación. Siguiendo la mirada estereotipada de ambos polos, diríamos que la primera es de menor valía y prestigio que la segunda y sólo la investigación es digna del reconocimiento de las casas de altos estudios, mientras la extensión es el voluntariado más sofisticado; nunca el extensionista tendrá el prestigio y galardón de las instituciones pero sí la gloria que conlleva el abrazo del pueblo, cediendo al investigador los aplausos del auditorio de colegas y la condecoración del yerto bronce.

La superación de dichos extremos no implica sólo desterrar las concepciones prejuiciosas hacia ambas áreas sino integrarlas a partir de una teoría dialéctica del conocimiento. Específicamente requiere implementar, desarrollar y ejercitar el proceso de acciónreflexión-acción y/o práctica-teoría-práctica de los grupos, movimientos, organizaciones en orden al logro de subvertir la realidad objetiva en la que se encuentran, siendo ellos partícipes en las acciones, reflexiones e interpretaciones de la realidad y sus comportamientos, influyendo en los aspectos objetivos pero también en la carga subjetiva inherente al proceso.

La propuesta dialéctica abordada en el presente artículo parte de la práctica, del territorio, de la realidad; dicho de otro modo, práctica- teoría-práctica; una teoría que surge a través del desarrollo de un proceso estructurado de abstracción para explicar su sentido, un pasaje de las observaciones externas a las causas internas; de la esencia a la contingencia, de lo dado a la historia, pero sin relativismos ni vacilación. Adquiriendo un horizonte integrador de la realidad, se articulan dinámicamente los elementos que la integran en una unidad compleja y paradójica que constituye la realidad cotidiana; un sueño por alcanzar pero creando lo deseado. Cuestionando la lógica causal de los eventos y su dinámica interna se implementa un paradigma crítico y creador del hacer social en el cual se incorporan las creencias culturales a la teorización, anclando los constructos a los procesos organizacionales, comunicacionales y educativos; la realidad jamás dejará de ser dinámica y el pensamiento siempre deberá estar despojado de dogmatismos; ambos son la dialéctica primordial.

En síntesis, el curso de extensión universitaria fue un espacio de encuentro de dos ámbitos, extensión e investigación, que nunca deben estar desvinculados sino asociados en un diálogo de enriquecimiento mutuo y fraternidad cuya única meta es contribuir y fomentar la transformación social, pues conocimiento y acción son el alma de una sociedad protagonista de su propia revolución; la ESS anhela y lucha por el mismo sueño.

\section{Referencias bibliográficas}

Fals Borda, O. (1993). Vigencias de las utopías en América Latina (193-206). En Osorio Vargas, J. y Weinstein, L. El corazón del arco iris: lecturas sobre nuevos paradigmas en educación y desarrollo. Santiago de Chile: CEAAL.

Ferrada, D. y Flecha, R. (2008). El modelo dialógico de la pedagogía: un aporte desde las experiencias de comunidades de aprendizaje. En Estudios Pedagógicos, 24 (1), 41-61.

Martínez, M. (2006). La investigación cualitativa (síntesis conceptual). En Revista IIPSI, 9 (1), 123-146

Montero, I. y León, O.G. (2007). Guía para nombrar los estudios de investigación en Psicología. En International Journal of Clinical and Health Psychology, 7 (3), 847-862.
Petit, L.; Baliño, G.; Iglesias, M.F. y Garo, M. (2014). Tensiones y desafíos para la construcción de otra economía: reflexiones desde el Diploma de Operador Socioeducativo en Economía Social y Solidaria. En: ExT: Revista de Extensión de la Universidad Nacional de Córdoba (Revista electrónica), (5), 1-18. Disponible en: http://revistas.unc.edu.ar/index.php/ext (recuperado el 5de mayo de 2015).

Petit, L. y Roura M.R. (2013). Las Comunidades de Aprendizaje como dispositivo del Diploma de Operador Socioeducativo en Economía Social y Solidaria, desde la perspectiva docente. En Hologramática: Revista Académica de la Facultad de Ciencias Sociales UNLZ (Revista electrónica), 19 (2), 61-76. Disponible en: http:// www.cienciared.com.ar/ra/usr/3/1480/hologramatica_n19v2pp61_76.pdf (recuperado e, 5 de mayo de 2015). 